\title{
An apology or absolution for cerebral near-infrared spectroscopy: Can we get on the same wavelength?
}

\author{
Rohit Rao, MD, ${ }^{\mathrm{a}}$ John J. Nigro, MD, ${ }^{\mathrm{b}}$ and Tara Karamlou, MD
}

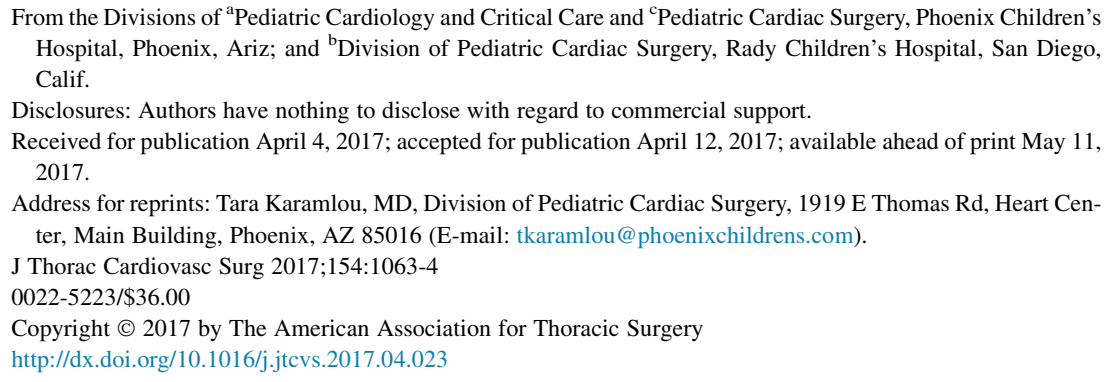

In this issue of the Journal, Rescoe and colleagues ${ }^{1}$ have published a retrospective single-institution study investigating the sensitivity and specificity of near-infrared spectroscopic (NIRS) monitoring in the cerebral bed in detecting low superior vena cava (SVC) central venous saturation $\left(\mathrm{ScVO}_{2}\right)$ in the setting of postoperative patients who have undergone a Norwood operation. Rescoe and colleagues ${ }^{1}$ studied 73 neonates and used 520 time-matched pairs, although the elapsed interval from time zero (removal of the aortic crossclamp) is never specified. Given that cerebral NIRS is highly weighted toward cerebral venous oxygen saturation but does contain arterial "contamination" (roughly $70 \%$ venous and $30 \%$ arterial), Rescoe and colleagues ${ }^{1}$ used a correction factor to account for this confounder. On the basis of their analysis of the matchedpairs data, they concluded that measurement of cerebral regional saturation $\left(\mathrm{rSO}_{2}\right)$ is insensitive to detect low $\mathrm{SCVO}_{2}$; however, correction for arterial contamination reduces a meaningful mean bias. Moreover, they reported that although values of cerebral $\mathrm{rSO}_{2}$ of less than 30 are specific for low $\mathrm{ScvO}_{2}$, that higher $\mathrm{rSO}_{2}$ values near or above 50 should not be used as reassurance of adequate $\mathrm{ScvO}_{2}$ in isolation.

What are we, as a specialty of congenital care providers, to make of these conclusions, when at first glance these data call the utility of NIRS into question? Let's start with a brief primer on cerebral NIRS and the literature that supports its widespread use. The current evidence in favor of NIRS monitoring in human studies during the past decade is consistent with class II, level B recommendations according to the American Heart Association and the American College of Cardiology Task Force on Practice Guidelines. ${ }^{2}$ NIRS is a noninvasive venous-weighted oximetric measurement that estimates the regional oxygen supply-demand relationship. Regional tissue beds have specific organ-centric oxygen extraction ratios and differing circulatory control mechanisms. ${ }^{3}$ Continuous $\mathrm{rSO}_{2}$ measurements reflect real-time changes in either sample bed.

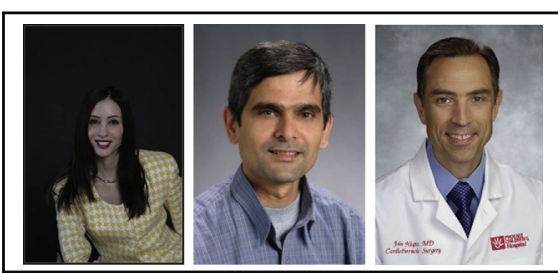

Left to right. Tara Karamlou, MD, Rohit Rao, MD, and John J. Nigro, MD

\section{Central Message}

Cerebral near-infrared spectroscopic monitoring should be interpreted with full knowledge of the limitations of the technology.

See Article page 1056.

metabolic demand or oxygen supply in the sampled region. In essence, NIRS monitors the oxygen economy in the

When using the NIRS technology, the depth of the optical field that is analyzed is approximately half the light sourcedetector distance on the sensor, which is $4 \mathrm{~cm}$ in most widely used devices. This source-detector distance and the superficial signal subtraction algorithm enable the device to estimate the $\mathrm{rSO}_{2}$ in a tissue sample about $1 \mathrm{~cm}^{3}$ in volume and about 2 to $3 \mathrm{~cm}$ beneath the sensor. When placed on the scalp, the monitor detects signal from the part of the frontal cortex in the range mentioned. Intravascular optical catheters have been placed in the SVCs of patients after the Norwood operation to guide therapy by analyzing the ratio of pulmonary to systemic perfusion with continuous data acquisition. ${ }^{4}$ It is not surprising that the venous saturation measurements from the SVC are lower than the $\mathrm{rSO}_{2}$ measured from a $1-\mathrm{cm}^{3}$ volume of brain tissue in the frontal cortex. The venous sample in the SVC is derived from the summation of venous saturation from the entire brain and the upper body, and perhaps even the lower body in patients with interrupted inferior vena cava and azygous continuation. In addition, the $\mathrm{rSO}_{2}$ is a venousweighted saturation with a small arterial component in the measurement (20\%-25\%), and the true venous saturation cannot be expected to be the same as the $\mathrm{rSO}_{2}$ from the limited sampling field. ${ }^{5}$ Typically, 2 sites are monitored by NIRS. This enables one to monitor organ beds that have differing hierarchic controls for flow metabolism coupling and thus provide a window on distribution of cardiac output. ${ }^{4}$ Indeed, multisite NIRS monitoring is 
becoming increasingly frequent in many pediatric cardiac intensive care units, because integration of multisite data and pattern recognition can provide complementary information. For instance, a reversal of the normal ratio of cerebral $\mathrm{rSO}_{2}$ to somatic $\mathrm{rSO}_{2}$ (where the somatic $\mathrm{rSO}_{2}$ is usually 10 points higher than cerebral $\mathrm{rSO}_{2}$ ) may be evident in shock states, in which there is relative reduction in somatic blood flow to redistribute blood flow preferentially to the brain.

The assessment of the true mixed venous saturation measurement is not possible in single-ventricle physiology because of the mixing lesions. If one were to obtain the venous weighted saturations from the inferior vena cava and SVC, however, then a close estimate could be made. A global quasi-mixed venous saturation can be reasonably approximated by NIRS models that include somatic and cerebral sites. ${ }^{5}$

Given this background information regarding NIRS, the limitations and confounders inherent in such a noninvasive metric of cerebral blood flow are evident. Rescoe and colleagues $^{1}$ have attempted to correct for one factor that reduces the accuracy of $\mathrm{rSO}_{2}$ as an estimate of $\mathrm{ScvO}_{2}$ (the arterial component of the sample in the $\mathrm{rSO}_{2}$ measurement). One must wonder, however, whether the differences in the $\mathrm{rSO}_{2}$ and the $\mathrm{ScvO}_{2}$ can be explained by other factors as stated previously. Perhaps more concerning given the presumed utility of NIRS as a trending monitor in the perioperative phase, were the data in which changes in cerebral $\mathrm{rSO}_{2}$ were found to be poorly correlated with changes in $\mathrm{ScVO}_{2}$. Although it is readily understood that absolute NIRS values are unreliable because of the inability to determine photon path length with available technology, the report of Rescoe and colleagues ${ }^{1}$ would suggest that trend monitoring of $\mathrm{rSO}_{2}$ values, though ostensibly valuable in demonstrating changes from baseline $\mathrm{rSO}_{2}$, may not reflect corresponding trends in other metrics that assess the adequacy of cerebral blood flow. In short, our synthesis of the article by Rescoe and colleagues ${ }^{1}$ is that cerebral $\mathrm{rSO}_{2}$ should be interpreted in the context of the other simultaneous hemodynamic and clinical metrics assessed in the postoperative Norwood setting and is therefore an adjunct rather than an absolute.

The future of pediatric cardiac intensive care is in increasingly focused on noninvasive technology and assimilation and analysis of real-time hemodynamic and clinical data by computers. The article by Rescoe and colleagues ${ }^{1}$ elucidates some of the problems inherent in the current technology and highlights the importance of further innovations to increase reliability and accuracy of the currently available instruments.

\section{References}

1. Rescoe E, Tang X, Perry DA, Sleeper LA, DiNardo JA, Kussman BD, et al. Cerebral near infrared spectroscopy insensitively detects low cerebral venous oxygen saturations after stage 1 palliation. J Thorac Cardiovasc Surg. 2017; 154:1056-62.

2. Tweddell JS, Ghanayem NS, Mussatto KA, Mitchell ME, Lamers LJ, Musa NL, et al. Mixed venous oxygen saturation monitoring after stage 1 palliation for hypoplastic left heart syndrome. Ann Thorac Surg. 2007;84: 1301-11.

3. Hoffman GM, Ghanayem NS, Kampine JM, Berger S, Mussatto KA, Litwin SB, et al. Venous saturation and the anaerobic threshold in neonates after the Norwood procedure for hypoplastic left heart syndrome. Ann Thorac Surg. 2000;70: 1515-20; discussion 1521.

4. Rao RP, Danduran MJ, Loomba RS, Dixon JE, Hoffman GM. Near-infrared spectroscopic monitoring during cardiopulmonary exercise testing detects anaerobic threshold. Pediatr Cardiol. 2012;33:791-6.

5. Watzman HM, Kurth CD, Montenegro LM, Rome J, Steven JM, Nicolson SC. Arterial and venous contributions to near-infrared cerebral oximetry. Anesthesiology. 2000;93:947-53. 